\title{
KINETIC ENERGY AND ADDED MASS OF HYDRODYNAMICALLY INTERACTING GAS BUBBLES IN LIQUID
}

\author{
J.B.W. KOK \\ Department of Physics, Twente University of Technology, P.O. Box. 217, 7500 AE Enschede,
} The Netherlands

Received 20 August 1986

Revised manuscript received 23 June 1987

\begin{abstract}
By averaging the basic equations on microscale, expressions are derived for the effective added mass density and the kinetic energy density of a mixture of liquid and gas bubbles. Due to hydrodynamic interaction between the bubbles there appears to be a difference between the effective added mass density and the mass coefficient of the kinetic energy density due to relative motion of the phases. For spherical bubbles with velocities all equal, isotropic spatial distribution, gas fraction $\alpha$ and liquid density $\rho_{1}$, the effective added mass density and the mass coefficient of the kinetic energy density (due to relative motion) are calculated. They are respectively $0.5 \alpha(1+$ $3.324 \alpha) \rho_{1}$ and $0.5 \alpha(1-0.676 \alpha) \rho_{1}$ and show good agreement with results in the literature.
\end{abstract}

\section{Introduction}

An expression for the added mass of a solitary massless body moving through an unbounded perfect liquid was derived by Kelvin. The added mass is found to be equal to the mass coefficient in the kinetic energy of the liquid in a frame moving with the (uniform) liquid velocity at infinity. In case there is a cloud of massless bodies in the liquid, Kelvin's approach for the calculation of the added mass can be extrapolated for the cloud as a whole.

In section 2 expressions are derived for the effective added mass density and the kinetic energy density of a mixture of liquid and gas bubbles, at low gas concentrations. In the literature it is sometimes assumed, or taken for granted, that the equality between the mass coefficient in the kinetic energy (due to relative motion) and the added mass, which exists in case of a solitary body, can be extended to mixtures. The calculation in section 2 shows that this equality does not exist at higher values of the concentration, when interactions have to be taken into account. Quantitative calculations for certain pair probability functions are carried out in section 3 . They are discussed in section 4 in connection with other results obtained in the literature. 


\section{On the relation between the effective added mass and kinetic energy density of a mixture of liquid and gas bubbles}

Consider an unbounded perfect liquid with density $\rho_{1}$ through which a solitary gas bubble moves. The bubble can be regarded as massless and incompressible. It will be assumed that the velocity field is uniform in absence of the bubble. Therefore a frame of reference can be chosen that moves with the liquid velocity at infinity. In this frame the liquid is at rest at infinity and the potential describing the velocity field due to the motion of the bubble is $\phi$. The impulse $I$ of the liquid is then defined by Kelvin $\left(\mathrm{Lamb}^{8}\right)$, p. 161) as

$$
I=-\int_{S} \rho_{1} \phi \mathrm{d} \boldsymbol{A},
$$

where the integration is over the surface $S$ of the bubble and $\mathrm{d} A$ is a surface element directed normal to the bubble surface.

If the bubble is spherical, or its motion is along an axis of symmetry then its added mass $\rho_{1} M$ is a scalar and defined by the expression (Batchelor ${ }^{3}$ ), p. 408)

$$
\rho_{1} M w=I
$$

with relative bubble velocity $\boldsymbol{w}$.

The kinetic energy of the liquid in a frame moving with the liquid velocity at infinity can be shown to be (Batchelor ${ }^{3}$ ), p. 403)

$$
T=-\frac{1}{2} \boldsymbol{w} \cdot \int_{S} \rho_{1} \phi \mathrm{d} \boldsymbol{A} .
$$

Substituting eqs. (1) and (2) in (3), the expression for the kinetic energy with respect to a frame moving with the liquid velocity at infinity becomes

$$
T=\frac{1}{2} \rho_{1} M w \cdot w
$$

For the calculation of the effective added mass and the kinetic energy density of a mixture of liquid and massless identical gas bubbles the mixture is thought to be embedded in an unbounded volume of pure liquid. In fact the solitary bubble is now replaced by a cloud of bubbles. Again a frame of reference will be chosen that moves with the liquid velocity at infinity. In this frame the potential describing the velocity field due to the motion of the bubbles is $\phi$. Now we will look for expressions for the impulse and the added mass of the cloud of bubbles as a whole. 
The impulse can be derived by summing eq. (1) over the surfaces of the $N$ bubbles in the cloud:

$$
\boldsymbol{I}=-\sum_{i=1}^{N} \int_{S_{i}} \rho_{1} \phi \mathrm{d} \boldsymbol{A}_{i} .
$$

The possibility to do this was already recognized by Lamb ${ }^{8}$ ) (p. 162).

We make the number of bubbles $N$ and the enveloping volume $V$ of the cloud very large, while the number density $n=N / V$ remains small. In this case it is more useful to speak in terms of (for example) impulse density rather than impulse. Also it should be stressed that the velocity $w$ of the bubbles is relative to a frame fixed to the liquid at infinity. From continuity it follows then that the liquid velocity at infinity equals the volume velocity in the cloud. In fact a frame is chosen that moves with the volume velocity. In this frame the impulse density is defined as

$$
\boldsymbol{I}^{\prime}=\boldsymbol{I} / V
$$

In analogy with formula (3) we have for the kinetic energy density of the liquid in the above-mentioned frame

$$
T^{\prime}=\frac{T}{V}=-\frac{1}{V} \sum_{i=1}^{N} \frac{1}{2} w_{i} \cdot \int_{S_{i}} \rho_{1} \phi \mathrm{d} A_{i}
$$

For small number densities it is possible to approximate the eqs. (5) and (7) to a form which can be handled. By means of methods common in statistical mechanics (see Batchelor ${ }^{1,2}$ )) the sums in (6) and (7) can be approximated by

$$
\begin{aligned}
& \boldsymbol{I}^{\prime}=-n\left\langle\int_{S_{i}} \rho_{\mathbf{l}} \phi \mathrm{d} \boldsymbol{A}_{i}\right\rangle+\mathcal{O}\left(n^{s+1}\right), \\
& T^{\prime}=-\frac{n}{2}\left\langle\boldsymbol{w}_{i} \cdot \int_{S_{i}} \rho_{l} \phi \mathrm{d} \boldsymbol{A}_{i}\right\rangle+\mathcal{O}\left(n^{s+1}\right),
\end{aligned}
$$

with $s=1,2, \ldots$

In (8) and (9) the quantities between brackets are ensemble averaged over all possible configurations of $s$ interacting bubbles. As we are interested in the relation between the effective added mass density and the kinetic energy density of the mixture, an expression is needed for the former. The expression 
for the effective added mass density $\rho_{1} M^{*}$ defined by eq. (4.12) in Van Wijngaarden ${ }^{11}$ ) will be used here:

$$
\rho_{1} M^{*}\left\langle\boldsymbol{w}_{i}\right\rangle=\boldsymbol{I}^{\prime} .
$$

Following Batchelor ${ }^{2}$ ), p. 228, this definition is the analogue for mixtures of the isolated bubble added mass in eq. (2). In order to have (for simplicity) a scalar $M^{*}$, it will be assumed that the bubbles are moving along their axis of symmetry.

Using (8), (9) and (10) the kinetic energy density of the mixture, in a frame moving with the volume velocity, can be shown to be

$$
T^{\prime}=\frac{1}{2} \rho_{1} M^{*}\left\langle\boldsymbol{w}_{i}\right\rangle \cdot\left\langle\boldsymbol{w}_{i}\right\rangle
$$

on the condition that the following equation holds:

$$
\left\langle\boldsymbol{w}_{i} \cdot \int_{S_{i}} \rho_{1} \phi \mathrm{d} \boldsymbol{A}_{i}\right\rangle=\left\langle\boldsymbol{w}_{i}\right\rangle \cdot\left\langle\int_{S_{i}} \rho_{1} \phi \mathrm{d} \boldsymbol{A}_{i}\right\rangle .
$$

There are two circumstances in which eq. (12) holds. In the first place when the bubbles have equal velocities, because then $\boldsymbol{w}_{i}=\left\langle\boldsymbol{w}_{i}\right\rangle$. The second case occurs when interactions are not taken into account. In all other events (12) does not hold and we must conclude that, in an approximation in which interactions are taken into account, the effective added mass density and the mass coefficient in the kinetic energy density will be different.

Lhuillier ${ }^{9}$ ) and Geurst ${ }^{6}$ ) attack the problem of formulation of equations of motion for a bubbly flow by starting with a Lagrangian. Both authors write the kinetic energy density in the Lagrangian, for the case of spherical bubbles, as

$$
T^{\prime}=\frac{1}{2} \rho_{\mathrm{g}} \alpha\left(\left|\boldsymbol{U}_{\mathrm{g}}\right|\right)^{2}+\frac{1}{2} \rho_{1}(1-\alpha)\left(\left|\boldsymbol{U}_{1}\right|\right)^{2}+\frac{1}{2} \rho_{1} f(\alpha)\left(\left|\boldsymbol{U}_{\mathrm{g}}-\boldsymbol{U}_{\mathrm{l}}\right|\right)^{2},
$$

with average liquid velocity $U_{1}$, average gas velocity $U_{\mathrm{g}}$, gas density $\rho_{\mathrm{g}}$, liquid density $\rho_{1}$ and gas concentration by volume $\alpha$. This is the kinetic energy density observed in a frame at rest. Lhuillier leaves $f(\alpha)$ as it is. He only observes that in the limit $\alpha \rightarrow 0 f(\alpha)$ must tend to $0.5 \alpha$. This is correct, as we will see.

Geurst ${ }^{6}$ ), in his formulation of the kinetic energy density in a rest frame, identifies from the start $\rho_{1} f(\alpha)$ with the effective added mass density. In the lines above we formulated, eq. (9), the kinetic energy density in a frame moving with the volume velocity. In order to make a comparison possible, we write down also the kinetic energy density in a rest frame. With liquid velocity $\boldsymbol{u}$ in that frame, we have 


$$
T^{\prime}=\frac{1}{2} \rho_{1} \frac{1}{V} \int_{V_{1}} \boldsymbol{u} \cdot \boldsymbol{u} \mathrm{d} V=\frac{1}{2} \rho_{\mathrm{l}}(1-\alpha) \frac{1}{V_{1}} \int_{V_{\mathrm{t}}} \boldsymbol{u} \cdot \boldsymbol{u} \mathrm{d} V
$$

The integration takes place over the volume $V_{1}$ of the liquid phase in the averaging volume $V$. The local liquid velocity $\boldsymbol{u}$ in a frame at rest can be expressed as the sum $U_{0}+\boldsymbol{u}^{\prime}$. In this expression is $\boldsymbol{u}^{\prime}$ the local liquid velocity in a frame moving with the volume velocity $U_{0}$ of the mixture. Substitution of $\boldsymbol{U}_{0}+\boldsymbol{u}^{\prime}$ for $\boldsymbol{u}$ in (14) gives

$$
T^{\prime}=\frac{1}{2} \rho_{1}(1-\alpha)\left\{U_{0}^{2}+\frac{2}{V_{1}} \int_{V_{1}} \boldsymbol{u}^{\prime} \mathrm{d} V \cdot U_{0}\right\}+\frac{1}{2} \rho_{\mathbf{l}} \frac{1}{V} \int_{V_{1}} \boldsymbol{u}^{\prime} \cdot \boldsymbol{u}^{\prime} \mathrm{d} V
$$

This can be rewritten in terms of $\rho_{1}, \alpha, \boldsymbol{U}_{1}, \boldsymbol{U}_{\mathrm{g}}$ and $\boldsymbol{u}^{\prime}$ with

$$
\frac{1}{V_{1}} \int_{V_{1}} \boldsymbol{u}^{\prime} \mathrm{d} \boldsymbol{V}=\boldsymbol{U}_{1}-\boldsymbol{U}_{0}
$$

and

$$
\boldsymbol{U}_{0}=\boldsymbol{U}_{\mathrm{l}}+\alpha\left(\boldsymbol{U}_{\mathrm{g}}-\boldsymbol{U}_{1}\right)
$$

to give for the kinetic energy density of the liquid phase in a frame at rest

$$
T^{\prime}=\frac{1}{2} \rho_{1}(1-\alpha) U_{1}^{2}-\frac{1}{2} \rho_{1}(1-\alpha) \alpha^{2}\left(\left|U_{\mathrm{g}}-U_{1}\right|\right)^{2}+\frac{1}{2} \rho_{1} \frac{1}{V} \int_{V_{1}} \boldsymbol{u}^{\prime} \cdot \boldsymbol{u}^{\prime} \mathrm{d} \boldsymbol{V}
$$

The third term on the right-hand side of (18) is recognized as the kinetic energy density of the liquid in a frame moving with the volume velocity of the mixture. This can be written as

$$
T^{\prime}=\frac{1}{2} \rho_{1} k(\alpha)\left(\left|\boldsymbol{U}_{\mathrm{g}}-\boldsymbol{U}_{0}\right|\right)^{?}
$$

It is assumed that the bubbles are axially symmetric and moving along their axis of symmetry. For non-interacting bubbles $k(\alpha)$ is then $0.5 M_{1} \alpha+\mathscr{O}\left(\alpha^{2}\right)$, with real constant $M_{1}$. When interaction between the bubbles is taken into account terms of higher order in $\alpha$ have to be added. In fact $k(\alpha)$ is expanded for small $\alpha$ in a Taylor series around the non-interaction value. The $s$ th order term in the expansion is the contribution to $k(\alpha)$ due to the interaction between $s$ bubbles on microscale. Thus the coefficient $k(\alpha)$ can be expressed as 


$$
k(\alpha)=0.5 M_{1} \alpha\left(1+M_{2} \alpha+M_{3} \alpha^{2}+\cdots+M_{s} \alpha^{s-1}\right)+\mathscr{O}\left(\alpha^{s+1}\right),
$$

with real constants $M_{s}$. It follows then from (13) and (17) to (20) that for $s=3$, $f(\alpha)$ is given by

$$
\begin{aligned}
f(\alpha)= & 0.5 M_{1} \alpha\left[1-\frac{M_{1}\left(2-M_{2}\right)+2}{M_{1}} \alpha+\frac{M_{1}\left(1-2 M_{2}+M_{3}\right)+2}{M_{1}} \alpha^{2}\right] \\
& +\mathscr{O}\left(\alpha^{4}\right) .
\end{aligned}
$$

The values of the constants $M_{s}$ have to be determined by evaluation of formula (9).

From eq. (12) the conclusion was drawn that there exists an equality between the mass coefficient $\rho_{1} \mathrm{k}(\alpha)$ in the kinetic energy density in a frame moving with the volume velocity, and the effective added mass density $\rho_{1} M^{*}(\alpha)$ in only two cases: for $\alpha \rightarrow 0$, i.c. if bubble interaction is neglected, or for $\boldsymbol{w}_{i}=\left\langle\boldsymbol{w}_{i}\right\rangle$. With this result and eqs. (20) and (21) we see now that the mass coefficient $\rho_{1} f(\alpha)$ in the kinetic energy density in a frame at rest equals the effective added mass density $\rho_{1} M^{*}$ only in one case: for $\alpha \rightarrow 0$, when bubble interaction is not taken into account.

\section{Calculation of the kinetic energy density and effective added mass of a mixture of liquid and hydrodynamically interacting gas bubbles}

In this section expression (9) will be evaluated for a configuration of two spherical bubbles $(s=2)$. If $s=2$ every bubble is thought to interact hydrodynamically with one other bubble, and (9) is

$$
\left\langle-0.5 \rho_{1} n w_{i} \cdot \int_{S_{i}} \Phi \mathrm{d} A_{i}\right\rangle+\mathscr{O}\left(\alpha^{3}\right) .
$$

In order to carry out the averaging process in (22), a pair probability density function is needed. A probability density function will be used which is factorized in a velocity distribution function and a configurational distribution function. With regard to the velocity distribution it will be assumed that all bubbles move with the average gas velocity $\boldsymbol{U}_{\mathrm{g}}$. Since $\boldsymbol{w}_{i}=\left\langle\boldsymbol{w}_{i}\right\rangle=\boldsymbol{w}=\boldsymbol{U}_{\mathrm{g}}-\boldsymbol{U}_{0}$ the calculated mass coefficient of the kinetic encrgy density, in frame moving with $U_{0}$, and the effective added mass density will be identical.

The potential $\Phi$ for two spherical bubbles with equal radii $a$, separation $s$ and velocities $\boldsymbol{w}$ can be found in Biesheuvel ${ }^{5}$ ). He employed a rectangular 
co-ordinate system $(x, y, z)$ with origin coinciding with the instantaneous position of bubble 1 , and $x$-axis directed along the line of the centres. Also were used two systems of spherical polar co-ordinates $\left(r_{i}, \theta_{i}, \phi_{i}\right)$ with origins at the centres of the bubbles. With respect to the centre of bubble 1 the potential $\Phi$ is then

$$
\begin{aligned}
\Phi= & \sum_{n=1}^{\infty}\left(\begin{array}{c}
a \\
r_{1}
\end{array}\right)^{n+1} a\left\{w_{x} g_{0 n} P_{n}^{0}\left(\cos \theta_{1}\right)+w_{y} g_{1 n} P_{n}^{1}\left(\cos \theta_{1}\right) \cos \phi_{1}\right. \\
& \left.+w_{z} g_{1 n} P_{n}^{1}\left(\cos \theta_{1}\right) \sin \phi_{1}\right\} \\
& +\sum_{n=1}^{\infty}\left(\frac{a}{s}\right)^{n+1} a\left\{w_{x} g_{0 n} \sum_{q=0}^{\infty}\left(\begin{array}{c}
n+q \\
q
\end{array}\right)\left(\frac{r_{1}}{s}\right)^{q} P_{q}^{0}\left(\cos \theta_{1}\right)\right. \\
& -w_{y} g_{1 n} \sum_{q=1}^{\infty}\left(\begin{array}{c}
n+q \\
q+1
\end{array}\right)\left(\frac{r_{1}}{s}\right)^{q} P_{q}^{1}\left(\cos \theta_{1}\right) \cos \phi_{1} \\
& \left.-w_{z} g_{1 n} \sum_{q=1}^{\infty}\left(\begin{array}{c}
n+q \\
q+1
\end{array}\right)\left(\frac{r_{1}}{s}\right)^{q} P_{q}^{1}\left(\cos \theta_{1}\right) \sin \phi_{1}\right\},
\end{aligned}
$$

with

$$
\begin{aligned}
& g_{m n}=0.5 \sum_{p=0}^{\infty} K_{m n p}\left(\frac{a}{s}\right)^{p}, \\
& K_{m n 0}=(-1)^{m} \delta_{1 n}, \\
& K_{m n p}=(-1)^{m-1} \frac{n}{n+1} \sum_{q=1}^{0.5(p-n-3)}\left(\begin{array}{c}
n+q \\
n+m
\end{array}\right) K_{m q(p-n-q-1)}, \quad p \geqslant 1, \\
& \delta_{1 n}= \begin{cases}0 & \text { for } n \neq 1, \\
1 & \text { for } n=1,\end{cases}
\end{aligned}
$$

$P_{n}^{i}\left(\cos \theta_{1}\right)$ are Tesseral harmonics according to the definition of Hobson $\left.{ }^{7}\right)$.

The surface integral in (22) can be evaluated after substitution of (23) and making use of several orthogonality properties of Tesseral harmonics. After some calculations the quantity between brackets in (22) becomes for bubble 1

$$
0.5 \rho_{1} \alpha\left\{\left(\begin{array}{c}
w_{x} g_{01} \\
-w_{y} g_{11} \\
w_{z} g_{11}
\end{array}\right)-\sum_{j=1}^{\infty}\left(\frac{a}{s}\right)^{j+2}\left(\begin{array}{c}
\left(\begin{array}{c}
j+1 \\
1
\end{array}\right) w_{x} g_{0 j} \\
\left(\begin{array}{c}
+1 \\
2
\end{array}\right) w_{y} g_{1 j} \\
\left(\begin{array}{c}
+1 \\
2
\end{array}\right) w_{z} \boldsymbol{g}_{1 j}
\end{array}\right)\right\} \cdot \boldsymbol{w} .
$$

The summations over $j$ can be removed using eq. $\mathrm{A}(2)$ in Van Wijngaarden ${ }^{11}$ ) (or Biesheuvel ${ }^{5}$ ), eq. (3.4)): 


$$
\sum_{j=m}^{\infty}\left(\begin{array}{c}
j+1 \\
m+1
\end{array}\right) g_{m j}\left(\frac{a}{s}\right)^{j+2}=1+2 g_{m 1}(-1)^{m-1}
$$

Expression (25) is then simplified to

$$
0.5 \rho_{1} \alpha\left(\begin{array}{l}
w_{x}\left(-1+3 g_{01}\right) \\
w_{y}\left(-1-3 g_{11}\right) \\
w_{z}\left(-1-3 g_{11}\right)
\end{array}\right) \cdot w
$$

Using (24) and the relations for the coefficients $K_{m n p}$, expression (27) can be rewritten as

$$
0.5 \rho_{1}(0.5 \alpha)\left\{\boldsymbol{w} \cdot \boldsymbol{w}-3 \boldsymbol{u}(\boldsymbol{b}, \boldsymbol{b}+\boldsymbol{s}) \cdot \boldsymbol{w}+3 \sum_{p=6}^{\infty}\left(\frac{a}{s}\right)^{p}\left(\begin{array}{l}
w_{x} K_{01 p} \\
-w_{y} K_{11 p} \\
-w_{z} K_{11 p}
\end{array}\right) \cdot \boldsymbol{w}\right\}
$$

In $(28) \boldsymbol{u}(\boldsymbol{b}, \boldsymbol{b}+\boldsymbol{s})$ is the velocity at $\boldsymbol{b}$ in the mixture due to a bubble with velocity $\boldsymbol{w}$ at $\boldsymbol{b}+\boldsymbol{s}$ :

$$
\boldsymbol{u}(\boldsymbol{b}, \boldsymbol{b}+\boldsymbol{s})=0.5\left(\frac{a}{s}\right)^{3}\left[2 w_{x},-w_{y},-w_{z}\right]
$$

Expression (28) has now to be averaged over all possible configurations of the ensemble. This will be done the way Van Wijngaarden $\left.{ }^{11}\right)$ demonstrated. $P\left(C_{N}\right)$ is the conditional probability distribution of a configuration of $N$ bubbles and $P\left(C_{N} \mid \boldsymbol{b}\right)$ is the conditional probability distribution function. The latter is the probability distribution given there is a bubble in $\boldsymbol{b} . P\left(C_{N}\right)$ and $P\left(C_{N} \mid \boldsymbol{b}\right)$ are normalized such that

$$
\int P\left(C_{N}\right) \mathrm{d} C_{N}=\int P\left(C_{N} \mid \boldsymbol{b}\right) \mathrm{d} C_{N}=N !
$$

The average of the second term between the braces in (28) is then given by

$$
-3\left\langle\boldsymbol{u}\left(\boldsymbol{b}, C_{N}\right) \cdot \boldsymbol{w}\right\rangle=-\boldsymbol{w} \cdot \frac{3}{N !} \int \boldsymbol{u}\left(\boldsymbol{b}, C_{N}\right) P\left(C_{N} \mid \boldsymbol{b}\right) \mathrm{d} C_{N}
$$

The integral in (31) is not absolutely convergent if the configuration is reduced to two bubbles. In order to overcome this difficulty a renormalisation technique due to Batchelor ${ }^{1}$ ) has to be used. This technique removes the convergence problem by subtracting from (31) an averaged quantity with a known value and an identical behaviour of its integrand for large separations of the bubbles. The quantity to be used here is 


$$
-\boldsymbol{w} \cdot \frac{3}{N !} \int \boldsymbol{u}\left(\boldsymbol{b}, C_{N}\right) P\left(C_{N}\right) \mathrm{d} C_{N}=0
$$

The integral in (32) is equal to the volume velocity. Since the volume velocity in a frame moving with the volume velocity of the mixture is zero, vanishes the integral in (32). Subtraction of (31) from (32) gives

$$
\left.3\left\langle\boldsymbol{u}\left(\boldsymbol{b}, C_{N}\right) \cdot \boldsymbol{w}\right)\right\rangle=\boldsymbol{w} \cdot \frac{3}{N !} \int \boldsymbol{u}\left(\boldsymbol{b}, C_{N}\right)\left(P\left(C_{N} \mid \boldsymbol{b}\right)-P\left(C_{N}\right)\right) \mathrm{d} C_{N} .
$$

This can be reduced to a two-bubble configuration without convergence problems. Now $P(\boldsymbol{b}+\boldsymbol{s} \mid \boldsymbol{b})$ and $P(\boldsymbol{b}+\boldsymbol{s})$ have to be specified. It will be assumed that the configurational distribution is completely random (analogous to Van Wijngaarden $\left.{ }^{l 1}\right)$ ):

$$
\begin{aligned}
& P(\boldsymbol{b}+s \mid b)= \begin{cases}0 & \text { for } s<2 a, \\
n & \text { for } s \geqslant 2 a,\end{cases} \\
& P(\boldsymbol{b}+s)=n \text { for all } s .
\end{aligned}
$$

Reducing (33) to a two-bubble configuration and using (34) the former becomes

$$
3\langle\boldsymbol{u}(\boldsymbol{b}, \boldsymbol{b}+\boldsymbol{s}) \cdot \boldsymbol{w}\rangle=-3 n \boldsymbol{w} \cdot \int_{s \leqq 2 a} u(b, b+s) \mathrm{d} s .
$$

In order to evaluate the integral the role of the bubbles is interchanged. Instead of $\boldsymbol{u}(\boldsymbol{b}, \boldsymbol{b}+\boldsymbol{s})$ is considered $\boldsymbol{u}(\boldsymbol{b}+\boldsymbol{s}, \boldsymbol{b})$. This is the velocity at $\boldsymbol{b}+\boldsymbol{s}$ when there is a bubble at $\boldsymbol{b}$. In the region $s \leqq a, u(b+s, b)$ equals $\boldsymbol{w}$. For $a<s \leqq 2 a, \boldsymbol{u}(\boldsymbol{b}+\boldsymbol{s}, \boldsymbol{b})$ is given by (29). This gives for (35)

$$
\begin{aligned}
3\langle\boldsymbol{u}(\boldsymbol{b}, \boldsymbol{b}+\boldsymbol{s}) \cdot \boldsymbol{w}\rangle= & -\boldsymbol{w} \cdot \boldsymbol{w} \int_{s \subseteq a} 3 n \mathrm{~d} s \\
& -3 \boldsymbol{w} \cdot \int_{a \leq s \leqq 2 a} 0.5 n a^{3} s^{-3}\left[2 w_{x},-w_{y},-w_{z}\right] \mathrm{d} s .
\end{aligned}
$$

As the first term equals $-3 \alpha w \cdot w$ and the second term vanishes, (36) becomes

$$
-3\langle u(b, b+s) \cdot w\rangle=3 \alpha w \cdot w
$$

The other terms between the braces in (28) can be reduced to two-bubble 
configurations without convergence problems. Averaging the third term, using spherical polar co-ordinates gives

$$
\begin{aligned}
& \sum_{p=6}^{\infty} n w^{2} \int_{s=2 a}^{\infty} \int_{\theta=0}^{\pi} 2 \pi \sin \theta s^{2}\left(K_{01 p} \cos ^{2} \theta-K_{11 p} \sin ^{2} \theta\right)\left(\frac{a}{s}\right)^{p} \mathrm{~d} \theta \mathrm{d} s \\
& \quad=\alpha w^{2} \sum_{p=6}^{\infty} \frac{K_{01 p}-2 K_{11 p}}{(p-3) 2^{(p-3)}}=0.1080 \alpha w \cdot w .
\end{aligned}
$$

Summation of the results in (37) and (38) and the first order term $\boldsymbol{w} \cdot \boldsymbol{w}$ gives, with the coefficient $0.5 \rho_{1}(0.5 \alpha)$, the ensemble average of (28):

$$
0.5 \rho_{1}(0.5 \alpha)(1+3.324 \alpha) \boldsymbol{w} \cdot \boldsymbol{w}+\mathscr{O}\left(\alpha^{3}\right)
$$

This is the kinetic energy density of the liquid in the mixture with respect to a frame moving with the volume velocity. Its mass coefficient $\rho_{1} k(\alpha)$ is equal to

$$
\rho_{1} k(\alpha)=0.5 \alpha(1+3.324 \alpha) \rho_{1}+\mathscr{O}\left(\alpha^{3}\right)
$$

As proved in section 2 for this velocity distribution the effective added mass density is also equal to (40).

Comparison with (20) shows that $M_{1}=1$ and $M_{2}=3.324$. From (21) it follows then that the mass coefficient $f(\alpha)$ in the kinetic energy density of the mixture in a frame at rest is

$$
f(\alpha)=0.5 \alpha(1-0.676 \alpha)+\mathscr{O}\left(\alpha^{3}\right)
$$

\section{Comparison with results in the literature}

In this section some results derived in the literature for the kinetic energy density and effective added mass density will be discussed and compared to the results obtained in this paper.

Van Wijngaarden ${ }^{11}$ ) calculated the effective added mass density of a dilute mixture of spherical gas bubbles randomly distributed in liquid just after instantaneous acceleration to a volume velocity $\boldsymbol{U}_{0}$. He obtained

$$
\rho_{1} M^{*}(\alpha)=0.5 \alpha(1+3.21 \alpha) \rho_{1}+\sigma\left(\alpha^{3}\right)
$$

After the acceleration the bubbles have an average velocity $3 U_{0}(1-2.14 \alpha)$ in a frame at rest (the values listed here are corrected values). As the average 
bubble velocity depends on $\alpha$ the individual bubble velocity cannot be constant with respect to the averaging process. For this reason eq. (12) does not hold and the effective added mass density (42) must differ from the associated mass coefficient $\rho_{1} k(\alpha)$ of the kinetic energy density for terms of second order in $\alpha$.

Biesheuvel $^{5}$ ) found the effective added mass density of a mixture of liquid and spherical gas bubbles, with random configurational and uniform velocity distribution, to be

$$
\rho_{1} M^{*}(\alpha)=0.5 \alpha(1+3.324 \alpha) \rho_{1}+\mathcal{O}\left(\alpha^{3}\right) .
$$

This is equal to the result (40) obtained in this paper for this case. The difference between (42) and (43) in the terms of order $\alpha^{2}$ is surprisingly small, considering the difference in velocity distribution. Apparently the velocity distribution does not have much influence on the effective added mass density. Also it can be observed that (43) is larger than (42). This agrees with the general theorem (Batchelor ${ }^{2}$ ), p. 231) which states that the effective transport parameters have a maximum when the local transport properties of the mixture are uniform.

Zuber $^{12}$ ) made the following estimate of the effective added mass density:

$$
\rho_{1} M^{*}(\alpha)=0.5 \alpha(1+3 \alpha) \rho_{1}+\mathcal{O}\left(\alpha^{3}\right) \text {. }
$$

Again this result does not differ much from (42) or (43). The reason for this is explained in Van Wijngaarden ${ }^{11}$ ).

Geurst $\left.^{6}\right)$ wrote the kinetic energy density as (13) and identified from the start $\rho_{1} f(\alpha)$ with the effective added mass density $\rho_{1} M^{*}(\alpha)$. Using variational calculus Geurst derived equations of motion and subsequently investigated the characteristics of these. They are real provided $f(\alpha)$ behaves in a specific way as a function of $\alpha$. He finds that marginal stability is ensured, for spherical bubbles, when (p. 252)

$$
f(\alpha)=0.5 \alpha(1-4 \alpha)+\mathscr{O}\left(\alpha^{3}\right)
$$

Geurst compares this with values for the effective added mass density obtained in the literature, for example (42). He comments on the fact that marginal stability requires a specific configuration with a view to (45).

However, as we have seen in section 2 , it is not $\rho_{1} f(\alpha)$ but $\rho_{1} k(\alpha)$ that can bc compared with the effective added mass density if the fluctuations in the bubble velocities are negligible. Using (21), Geurst would obtain a coefficient $k(\alpha)$ in (20) with a vanishing constant $M_{2}$ : 


$$
k(\alpha)=0.5 \alpha+\mathscr{O}\left(\alpha^{3}\right)
$$

This result is remarkable. In despite of the $\mathscr{O}\left(\alpha^{3}\right)$ accuracy, the second order terms in $\alpha$, due to interaction, are absent. Geurst also derived an expression for $f(\alpha)$ with an accuracy of $\mathscr{O}\left(\alpha^{4}\right)$ (p. 251). This expression is equal to (21) if the constants $M_{2}$ and $M_{3}$, describing interactional effects, vanish. A very interesting point is now revealed. Though Geurst does take interactional effects into account up to order $\alpha^{3}$ (which is one order higher than any other reference in the literature), he must conclude that his equations have marginal stability only if

$$
k(\alpha)=0.5 M_{1} \alpha+\mathscr{O}\left(\alpha^{4}\right) .
$$

In other words, there is marginal stability if $\rho_{1} k(\alpha)$ (e.g. the effective added mass density) equals for every order in $\alpha$ (ranging from 1 to 3 ) the value in the non-interaction case. This is in line with the fact that Geurst finds the gas concentration waves in the mixture to travel with the speed of the bubbles ( $p$. 253). Biesheuvel and Van Wijngaarden ${ }^{4}$ ) also found this result: $k(\alpha)=0.5 \alpha$ and real characteristic speeds equal to the bubble speed. But as they did not take interactions into account, their results are only valid up to first order in $\alpha$.

The last result for the kinetic energy density that will be discussed is that of Oshima $\left.{ }^{10}\right)$. He found for $f(\alpha)$,

$$
f(\alpha)=0.5 \alpha(1-\alpha)+\mathscr{O}\left(\alpha^{3}\right) .
$$

Comparison with (41) shows that there is only a small difference in the second order term in $\alpha$. This similarity can be explained as follows. Though Oshima does not refer to Zuber ${ }^{12}$ ) he uses the same (approximating) method, velocity distribution and configurational distribution. Their velocity distribution is such that all bubbles have the average gas velocity. Therefore (12) holds and the effective added mass density equals the mass coefficient $\rho_{l} k(\alpha)$. For this reason it should be expected that (44) and (48) yield the same result. Using (20), (21) and (48) it is confirmed that the effective added mass density of Zuber ${ }^{12}$ ) and the mass coefficient $\rho_{1} k(\alpha)$ of Oshima are identical.

\section{Conclusion}

There is a fundamental difference between the effective added mass density and the mass coefficient of the kinetic energy density due to relative motion, of a mixture of liquid and gas bubbles. Only if the fluctuations in the bubble 
velocities are negligible and with the help of a conversion formula a comparison is possible for terms of second and higher order in the gas fraction. For this velocity distribution and an isotropic spatial distribution the kinetic energy density has a negative dependance on the gas fraction $\alpha$ in second order terms, but does not vanish for $0<\alpha<1$. Stressing the difference the effective added mass density has a positive dependence on the gas fraction in the second order terms. The results agree well with the results in the literature.

\section{Acknowledgements}

The author would like to thank Prof. L. van Wijngaarden for drawing his attention to the relation between added mass and kinetic energy, and Dr. A. Biesheuvel for some useful discussions.

\section{References}

1) G.K. Batchelor and J.T. Green, J. Fluid Mech. 56 (1972) 401.

2) G.K. Batchelor, Ann. Rev. Fluid Mech. 6 (1974) 227.

3) G.K. Batchelor, An Introduction to Fluid Dynamics (Cambridge Univ. Press, Cambridge, 1970).

4) A. Biesheuvel and L. van Wijngaarden, J. Fluid Mech. 148 (1984) 301.

5) A. Biesheuvel, Pl.D. hhesis, Twente Univ. of Technology, Enschede (1984).

6) J.A. Geurst, Physica 129A (1985) 233.

7) E.W. Hobson, The Theory of Spherical and Ellipsoidal Harmonics (Cambridge Univ. Press, Cambridge, 1931).

8) H. Lamb, Hydrodynamics (Cambridge Univ. Press, Cambridge, 1957).

9) D. Lhuillier, Int. J. Multiphase Flow 11 (1985) 427.

10) N. Oshima, Bull. J.S.M.E. 22 (1979) 550.

11) L. van Wijngaarden, J. Fluid Mech. 77 (1976) 27.

12) N. Zuber, Chem. Eng. Sci. 19 (1964) 897. 